\title{
Recombinant anti-Müllerian hormone in the maturation medium improves the in vitro maturation of human immature (GV) oocytes after controlled ovarian hormonal stimulation
}

Jure Bedenk 1* $^{*}$, Tadeja Režen ${ }^{2}$, Taja Železnik Ramuta ${ }^{3}$, Nina Jančar ${ }^{4}$, Eda Vrtačnik Bokal $^{4}$, Ksenija Geršak $^{5}$ and Irma Virant Klun ${ }^{1}$

\begin{abstract}
Background: In vitro maturation (IVM) of oocytes is a laboratory method that allows the maturation of immature (GV) oocytes retrieved from patients enrolled in the in vitro fertilization (IVF) programme. However, this method is still sparsely researched and used in clinical practice, leading to suboptimal clinical results. Anti-Müllerian hormone (AMH) is an important hormone with known effects on human ovaries, especially on follicles (follicular cells) during folliculogenesis. In contrast, the effect of AMH on the human oocyte itself is unknown. Therefore, we wanted to determine whether human oocytes express AMH receptor 2 (AMHR2) for this hormone. Recombinant AMH was added to the IVM medium to determine whether it affected oocyte maturation.
\end{abstract}

Methods: In total, 247 human oocytes (171 immature and 76 mature) were collected from patients enrolled in the intracytoplasmic sperm injection (ICSI) programme who were aged 20 to 43 years and underwent a short antagonist protocol of ovarian stimulation. The expression of AMHR2 protein and AMHR2 gene was analysed in immature and mature oocytes. Additionally, maturation of GV oocytes was performed in vitro in different maturation media with or without added $\mathrm{AMH}$ to evaluate the effect of $\mathrm{AMH}$ on the oocyte maturation rate.

Results: Immunocytochemistry and confocal microscopy revealed that AMHR2 protein is expressed in both immature and mature human oocytes. AMHR2 was expressed in a spotted pattern throughout the whole oocyte. The IVM procedure revealed that $\mathrm{AMH}$ in maturation medium improved GV oocyte maturation in vitro, as all oocytes were successfully matured in maturation medium containing recombinant AMH only. Furthermore, antagonism between $\mathrm{AMH}$ and follicle-stimulating hormone (FSH) during the maturation process was observed, with fewer oocytes maturing when both $\mathrm{AMH}$ and FSH were added to the maturation medium. Finally, AMHR2 gene expression was found in immature and in vitro matured oocytes but absent in mature oocytes.

Conclusions: The positive AMHR2 protein and AMHR2 gene expression in human oocytes shows that AMH could directly act on human oocytes. This was further functionally confirmed by the IVM procedure. These findings suggest the potential clinical application of recombinant $\mathrm{AMH}$ to improve IVM of human oocytes in the future.

*Correspondence: jure.bedenk@kclj.si

${ }^{1}$ Clinical Research Centre, University Medical Centre Ljubljana,

1000 Ljubljana, Slovenia

Full list of author information is available at the end of the article permits use, sharing, adaptation, distribution and reproduction in any medium or format, as long as you give appropriate credit to the original author(s) and the source, provide a link to the Creative Commons licence, and indicate if changes were made. The images or other third party material in this article are included in the article's Creative Commons licence, unless indicated otherwise in a credit line to the material. If material is not included in the article's Creative Commons licence and your intended use is not permitted by statutory regulation or exceeds the permitted use, you will need to obtain permission directly from the copyright holder. To view a copy of this licence, visit http://creativecommons.org/licenses/by/4.0/. The Creative Commons Public Domain Dedication waiver (http://creativeco mmons.org/publicdomain/zero/1.0/) applies to the data made available in this article, unless otherwise stated in a credit line to the data. 
Keywords: Anti-Müllerian hormone, Anti-Müllerian hormone receptor 2, In vitro maturation, Human oocyte, Immunocytochemistry, Confocal microscopy, RT-qPCR

\section{Background}

Assisted reproductive technology (ART) has enabled many couples with fertility problems to conceive a child with the help of in vitro fertilization (IVF). Even though the technology and methods are evolving and becoming more sophisticated, there is still not much we can do regarding immature oocytes that are obtained by follicular aspiration after controlled ovarian hormonal stimulation. In most clinics, immature oocytes are discarded as part of daily practice, especially if they are at the germinal vesicle (GV; prophase I) stage, because they cannot be fertilized in the IVF programme. Fortunately, the percentage of immature oocytes obtained in the IVF programme is relatively low $[1,2]$. However, for certain patients, such as those with a low oocyte count ("poor responders") and oncology patients who undergo oocyte cryostorage for fertility preservation before chemo- and radiotherapy, these oocytes are very important and may be the only chance for embryo development and pregnancy achievement. Thus, in vitro maturation (IVM) of immature oocytes has been developed. In short, this is a laboratory method that involves collecting and culturing immature oocytes in maturation medium to obtain mature (MII) oocytes over time. These immature oocytes can be obtained from stimulated cycles after controlled ovarian hormonal stimulation, as well as from unstimulated cycles without hormonal stimulation in cancer patients, where time is of the essence and hormones pose a threat to the growth and spreading of the cancer, or in patients with polycystic ovary syndrome (PCOS), where the risk of ovarian hyperstimulation syndrome (OHSS) after hormonal stimulation is high [3, 4]). Afterwards, the oocytes are treated as mature (MII; metaphase II) oocytes and are fertilized in the IVF programme. As of 2021, IVM method is no longer treated as experimental, so it should have more clinical relevance [5]. To mature them in vitro, immature oocytes are cultured in complex maturation media with added hormones, such as follicle-stimulating hormone (FSH) and human chorionic gonadotropin (hCG), to increase the chances of their maturation [6]. The environment of the human ovary (ovarian niche) and in vivo maturation are still not fully understood (especially at the molecular level). Therefore, the human oocyte IVM procedure is not optimal at the moment, and only a relatively low percentage of oocytes manage to mature in this way [6]. Additionally, a low implantation rate and a high miscarriage rate are observed for IVM oocytes in comparison to conventional
IVF of oocytes that mature in the patient's ovaries after controlled ovarian hormonal stimulation [7].

The role of anti-Müllerian hormone (AMH) in oocyte maturation is not yet known. This dimeric glycoprotein $[8,9]$ is known mostly for its role in gonadal differentiation during embryonic development [10] and has an impact on ovarian function, particularly in terms of the growth and recruitment of follicles during folliculogenesis $[11,12]$. It is considered to be one of the best biomarkers for the evaluation of a patient's ovarian reserve [13-15], response to controlled ovarian stimulation [16] and the outcome of IVF, although the results of the latter vary $[17,18]$. It is also predicted that AMH can become a powerful tool for the diagnosis of certain ovarian illnesses, such as PCOS $[19,20]$, primary ovarian insufficiency (POI) [21], premature menopause [22] and gynaecological tumours [23-25]. The key receptor for $\mathrm{AMH}$ is anti-Müllerian hormone receptor 2 (AMHR2), which is expressed in testicular Sertoli [26-28] and Leydig cells [28-30], ovarian theca $[28,31]$ and granulosa cells [26-28], as well as in the prostate [32], breasts [33] and endometrium [34]. However, we were unable to find any data regarding the expression of AMHR2 on human oocytes in the literature, in comparison to certain animals, such as mice [35] and cattle [36].

Some scarce data in animal models have shown that AMH might act directly on oocytes and affect their maturation. In 2014, Zhang et al. [35] studied the effect of $\mathrm{AMH}$ on the maturation of immature mouse oocytes and proved that AMH has a positive effect on oocyte maturation in vitro. To our knowledge, this is the only study to date to test the effect of AMH on oocytes, even though the study was done in mice. AMH helps to keep ovarian follicles in an inactive state so that the ovarian reserve of the woman does not diminish too early $[37,38]$. In this study, we wanted to determine if the same is true for human oocytes, namely, if AMHR2 is expressed in immature and mature human oocytes and if the addition of $\mathrm{AMH}$ to the maturation medium affects oocyte maturation in vitro.

\section{Methods}

\section{Patients and sample collection}

This study included 247 (171 GV and 76 MII) oocytes from 96 patients enrolled in the IVF/intracytoplasmic sperm injection (ICSI) programme. Oocytes were retrieved from each patient after written informed consent was obtained at the University Medical Centre 
Ljubljana, Slovenia, from January 2019 to April 2021. The inclusion and exclusion criteria were different depending on the method used. For immunocytochemical staining, the only inclusion criteria were enrolment in IVF treatment (40 patients) and a patient age of 20 to 43 years. Patients with a low number of oocytes ( $<3$ total oocytes) attained by ultrasound-guided ovarian (follicular) aspiration or who were involved in the preimplantation genetic diagnosis (PGD) programme were excluded. On the other hand, for IVM and reverse-transcription quantitative real-time polymerase chain reaction (RT-qPCR) analysis, we used the following inclusion criteria: age from 20 to 38 years and ovarian stimulation using the short gonadotropin-releasing hormone $(\mathrm{GnRH})$ antagonist protocol (56 patients). The exclusion criteria were the same as those for the immunocytochemical analysis.

\section{Controlled ovarian hormonal stimulation}

Briefly, the $\mathrm{GnRH}$ antagonist protocol of ovarian stimulation was performed as follows: recombinant FSH stimulation was initiated on the 2nd day of the menstrual cycle. The daily dosage was adjusted individually depending on the patient status and ovarian response (between 1050 and 3000 IU; Gonal-F, Merck Europe B.V., The Netherlands or Bemfola, Gedeon Richter, Hungary or Pergoveris, Merck Europe B.V., The Netherlands). The patients were given $0.25 \mathrm{mg}$ of cetrorelix acetate (Cetrotide, Merck Europe B.V., The Netherlands) or $0.25 \mathrm{mg}$ of ganirelix (Orgalutran, MSD, USA) on Day 7 of the menstrual cycle, and the application continued daily until the day of the follicle trigger.

When a sufficient number of follicles reached a mean diameter of at least $17 \mathrm{~mm}$, patients were administered $250 \mu \mathrm{g}$ of recombinant hCG (Ovitrelle ${ }^{\circledR}$, Merck Europe B.V., The Netherlands). Oocytes were obtained using transvaginal ultrasound-guided needle aspiration of follicles 34 to $36 \mathrm{~h}$ after the application of recombinant hCG.

\section{Oocyte preparation}

After aspiration, cumulus-oocyte complexes (COCs) were transferred to flushing medium (Cooper Surgical, Denmark) for the removal of granulosa cells. After granulosa cell removal, oocytes were transferred to Universal IVF medium (Cooper Surgical, Denmark) and incubated for $1.5-2.5 \mathrm{~h}\left(37.0{ }^{\circ} \mathrm{C}\right.$ and $\left.6 \% \mathrm{CO}_{2}\right)$. Then, the oocytes were treated with hyaluronidase (SynVitro Hydase, Cooper Surgical, Denmark) for $20 \mathrm{~s}$ to remove the corona radiata cells. Thus, the cells were prepared for the following procedures.

\section{Immunocytochemical staining}

For immunocytochemical staining, 65 immature and 61 mature oocytes that did not fertilize after ICSI were immunostained using a modified protocol written by Virant Klun et al. [39]. In brief, the oocytes were first washed with PBS (Gibco, USA) and then fixed in $4 \%$ paraformaldehyde (Kemika, Croatia) for $8 \mathrm{~min}$. After fixation, oocytes were washed two times with phosphatebuffered saline (PBS) and permeabilized with $0.3 \%$ Triton X-100 for $10 \mathrm{~min}$. Then, oocytes were washed again with PBS. Unspecific binding of antibodies was blocked by incubating oocytes in 10\% foetal bovine serum (FBS) (Gibco, USA) in the dark for $20 \mathrm{~min}$. The detection of AMHR2 receptors was performed with a primary monoclonal mouse anti-AMHR2 antibody (Ab64762, Abcam, USA, 1:100 dilution) for $60 \mathrm{~min}$ in the dark. After this, oocytes were washed three times with PBS. For the visualization of the signal, secondary goat anti-mouse antibody was used (Alexa Fluor 488, Molecular Probes, USA, 1:200 dilution) for $30 \mathrm{~min}$ in the dark. Oocytes were washed in PBS one last time and transferred to an object glass with a drop of Vectashield Mounting Medium with DAPI (Vector, USA) for $15 \mathrm{~min}$ in the dark. The negative controls used similar conditions except for the substitution of primary antibodies with PBS.

Immunolabelling was observed under a fluorescence microscope (Nikon Eclipse E600, Nikon Inc., Japan) and confocal microscope (Zeiss LSM900, Carl Zeiss AG, Germany). The images obtained with the confocal microscope were further processed with Zeiss ZEN software (Blue edition, Carl Zeiss AG, Germany) to prepare 3D reconstructions.

Analysis of total cell fluorescence by fluorescence microscopy (Nikon Eclipse E600) was performed for the comparison of the expression level of AMHR2 protein using Fiji software [40]. Total cell fluorescence by fluorescence microscopy was calculated with the help of an adjusted formula found in Gavet and Pines [41]:

Corrected total cell fluorescence $(\mathrm{CTCF})=$ whole-cell fluorescence - (area selected $\mathrm{x}$ mean fluorescence of background readings).

For each image, we measured the fluorescence of the oocyte and the background (separately) in three repetitions. The average of each cell and its background was then used for the calculation of CTCF. CTCF is presented as average fluorescence \pm SD.

In addition, a western blot analysis was performed to test the specificity and validity of the anti-AMHR2 antibody used in this study (Ab64762, Abcam, USA, 1:100 dilution) compared to the new primary polyclonal rabbit anti-AMHR2 antibody of the same producer (Ab197148, Abcam, USA, 1:100 dilution). With this analysis, we have confirmed the specificity and validity of the antibody Ab64762. The comparative western blot image with the strongest band at $63 \mathrm{kDa}$ corresponding to AMHR2 detected by both antibodies is shown in Additional file 1 . 


\section{In vitro maturation of immature oocytes}

Ninety-one immature (GV) oocytes from 45 patients were matured using the MediCult IVM system (LAG and IVM medium, Cooper Surgical, Denmark). Briefly, after acquiring denuded GV oocytes without surrounding follicular cells, they were transferred to LAG medium for $2 \mathrm{~h}$. Later, cells were transferred to the 9-microwell culture dish (Vitrolife, Sweden) with one of four different IVM media applied: (1) IVM medium with the addition of recombinant AMH $100 \mathrm{ng} / \mathrm{mL}$ (1737-MS-010, R\&D Systems, USA; $n=15$ oocytes), (2) IVM medium with the addition of FSH $75 \mathrm{mIU} / \mathrm{mL}$ (Gonal-F, Merck Europe B.V., The Netherlands; $n=22$ oocytes) and hCG $100 \mathrm{mIU} / \mathrm{mL}$ (Ovitrelle, Merck Europe B.V., The Netherlands), (3) IVM medium with the addition of FSH $75 \mathrm{mIU} / \mathrm{mL}$, hCG $100 \mathrm{mIU} / \mathrm{mL}$ and AMH $100 \mathrm{ng} / \mathrm{mL}$ ( $n=42$ oocytes) and (4) IVM medium with no added hormones (control; $n=12$ oocytes). The cells were then incubated in a $\mathrm{CO}_{2}$ incubator $\left(37.0^{\circ} \mathrm{C}\right.$ and $6 \% \mathrm{CO}_{2}$ in air) with the PrimoVision time-lapse microscope (Vitrolife, Sweden) to follow the maturation of the oocyte. Oocytes were observed until their maturation (extrusion of a polar body) for a maximum of $28 \mathrm{~h}$. The time of GV breakdown and extrusion of the polar body (MII) was assessed using videos of maturation provided by time-lapse microscopy. All matured oocytes were frozen for later RT-qPCR analysis, while nonmatured oocytes were discarded.

\section{Single-cell RT-qPCR}

RT-qPCR analysis of the AMHR2 gene was performed using the Single Cell-to-CT kit (Ambion, USA). The kit includes solutions for single-cell lysis, reverse transcription, preamplification and real-time (q) PCR. TaqMan gene expression assays were used for gene expression analysis (Hs01086646-g1 for AMHR2, Hs00824723_m1 for $U B C$, Hs02758991-g1 for GAPDH and Hs99999909_ $\mathrm{m} 1$ for HPRT1, Thermo Fisher Scientific, USA). The $U B C, G A P D H$ and HPRT1 genes were used as reference genes. However, after the completion of RT-qPCR, only $U B C$ was used as an optimal reference gene for the obtained results, as chosen by Normfinder software [42]. qPCR was performed in a 384-well plate on a LightCycler 480 System (Roche Diagnostics GmbH, Germany) as described in the kit and TaqMan gene expression assays manual. The analysis was performed in 15 groups of oocytes with 5 oocytes per group; 9 groups consisted of oocytes that matured in vitro in three different IVM media with added hormones, 3 groups of immature (GV) oocytes and 3 groups of oocytes that were retrieved as mature oocytes by follicular aspiration but did not fertilize in the IVF programme. Additionally, each sample was analysed in triplicate. Differential expression analysis was performed using the comparative quantification algorithm $-\Delta \Delta$ Ct [43].

\section{Statistical analysis}

Statistical analysis was performed using SPSS software (IBM Corp., USA), GraphPad Prism software (GraphPad Software, USA) and Jamovi software [44]. The results obtained by immunocytochemistry and oocyte maturation in vitro were evaluated by Student's t-test or Fisher's exact test, while gene expression results obtained by RTqPCR analysis were evaluated by Kruskal-Wallis oneway analysis of variance. A $P$-value $<0.05$ was regarded as statistically significant.

\section{Results}

\section{Patient information}

There were no significant differences in the age, body mass index (BMI), serum FSH or luteinizing hormone (LH) levels among women donating oocytes for different analyses or for different oocyte groups for IVM with different maturation media and analysis of gene expression, as shown in Additional files 2 and 3.

\section{Detection of AMHR2 protein in immature and mature} human oocytes with fluorescence and confocal microscopy Of 126 oocytes (65 GV, $61 \mathrm{MII}$ ) collected for immunocytochemistry, 59 oocytes were successfully stained (33 GV, 26 MII; 2 GV oocytes from the GV pool were used as control). We observed the expression of AMHR2 protein on the surfaces and inside of both mature and immature oocytes (Figs. 1, 2, 3, Additional file 4). The differences in expression between these two groups of oocytes $\left(8.1 \times 10^{7} \pm 3.4 \times 10^{7} \quad\right.$ CTCF $\quad$ vs. $\quad 7.7 \times 10^{7} \pm 3.0 \times 10^{7}$ CTFC) were not statistically significant, as revealed by Student's t-test. The data is presented as average fluorescence $\pm S D$. However, we observed that AMHR2 protein was expressed in a spotted pattern throughout the whole cell (Fig. 3). Conversely, in the negative control, the expression of AMHR2 protein was almost not visible (Figs. $1 \mathrm{~g}$ and 1i).

Videos of AMHR2-positive oocytes stained by immunocytochemistry and recorded by confocal microscopy can be seen in the appendix (Additional file 5).

\section{Effect of AMH in medium on the maturation of GV oocytes in vitro}

After IVM, we observed a 100\% (15/15) maturation rate of oocytes that matured in the medium with added recombinant $\mathrm{AMH}$ only. In comparison, only a $68 \%$ $(15 / 22)$ maturation rate was achieved with the use of conventional IVM medium with added FSH and hCG. Next, the group of oocytes that were matured in IVM medium supplemented with all three hormones (FSH, 



Fig. 1 Expression of AMHR2 protein (green) in human oocytes, as revealed by immunocytochemistry. A-C Immature oocyte with germinal vesicle (GV). D-F Mature (MII) oocyte. G-I Control immature oocyte with germinal vesicle (primary antibody omitted). Images were taken with a fluorescence microscope at $400 \times$ magnification, and the scale bar represents $100 \mu \mathrm{m}$. AMHR2 protein is stained green, and genetic material is stained blue (DAPI)

hCG and $\mathrm{AMH}$ ) produced an even lower maturation rate of $36 \%(15 / 42)$. Last, in the control group of oocytes that were matured in IVM medium without hormones added, 25\% (3/12) of oocytes spontaneously matured in vitro (Fig. 4). All differences between groups, except between the last two groups, were statistically significant (Fisher's exact test, $P<0.05$ ), as shown in Fig. 4.

\section{Effect of AMH in IVM medium on the maturation dynamics of oocytes: GV breakdown and polar body release}

The mean times were $3.7 \mathrm{~h}$ to GV breakdown (MI stage) in oocytes and $20.5 \mathrm{~h}$ to polar body release (MII stage) (Fig. 5). The times needed to reach the MI stage were comparable among oocytes that were matured in the $\mathrm{AMH}, \mathrm{FSH}+\mathrm{hCG}$, and $\mathrm{FSH}+\mathrm{hCG}+\mathrm{AMH}$ media and were 3.5, 3.8 and $3.7 \mathrm{~h}$, respectively (Fig. 5). There was a tendency for the polar body to be released later if AMH was added to the IVM medium: 21.5 and 20.2 vs. $19.9 \mathrm{~h}$ (Fig. 5). However, the differences were not statistically significant (Student's t-test, $P<0.05$ ). In the control group of oocytes, both times were prolonged ( 4.2 and $22.2 \mathrm{~h}$ ) due to their slower spontaneous maturation.
Figure 6 shows images of oocytes maturing in vitro in various media $(\mathrm{AMH}, \mathrm{FSH}+\mathrm{hCG}$ and $\mathrm{FSH}+\mathrm{hCG}+\mathrm{AMH})$ under time-lapse microscopy.

\section{Expression of the $A M H R 2$ gene in immature and mature human oocytes}

RT-qPCR analysis confirmed the expression of the AMHR2 gene in immature and in vitro matured oocytes (Fig. 7). The expression of this gene was highest in oocytes that matured in medium with only added AMH and in immature (GV) oocytes. The expression of the $A M H R 2$ gene was lower in oocytes that matured in the presence of all three hormones (AMH, FSH and hCG). Finally, the lowest expression was observed in the conventional mode of IVM with FSH and hCG added to the maturation medium. Nevertheless, the differences were not statistically significant (Kruskal-Wallis one-way analysis of variance, $P<0.05)$.

Additionally, we observed that the AMHR2 gene was expressed in immature $(\mathrm{GV})$ and in vitro matured oocytes, while it was not expressed in oocytes that matured in vivo. The difference between immature oocytes and in vivo mature oocytes was statistically significant (Kruskal-Wallis one-way analysis of variance, $P<0.05)$. 


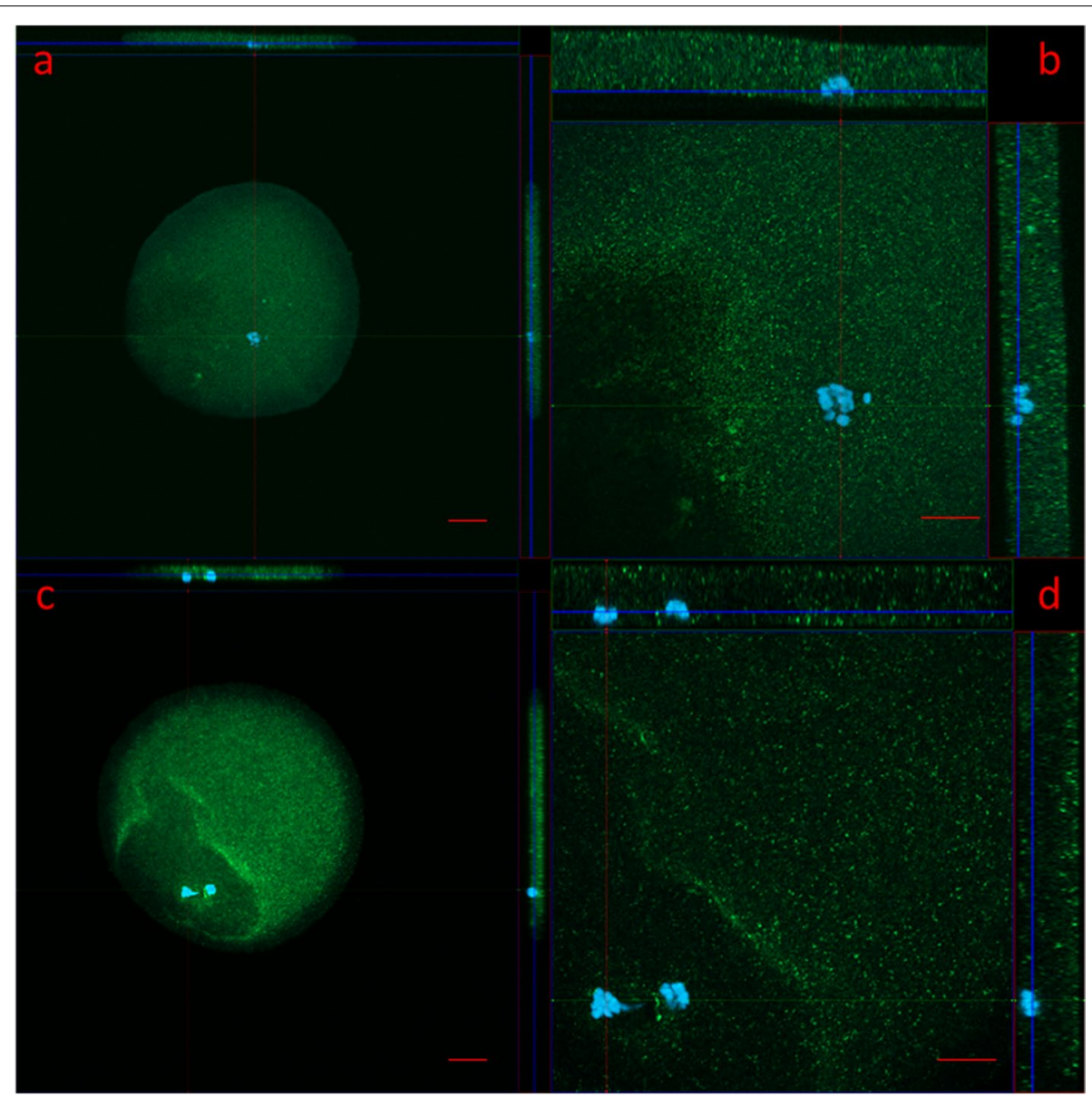

Fig. 2 Expression of AMHR2 protein (green) in human oocytes, as revealed by confocal microscopy. A Immature oocyte with a germinal vesicle (blue). B Enlarged section of the same immature oocyte with side horizontal cuts showing expression of AMHR2 protein throughout the oocyte. C Mature oocyte with a polar body. D Enlarged section of the same mature oocyte with side horizontal cuts showing expression of AMHR2 protein throughout the oocyte. Images were taken with a confocal microscope at $200 \times$ (images on the left, scale bar represents $20 \mu \mathrm{m}$ ) and $630 \times$ (images on the right, scale bar represents $10 \mu \mathrm{m}$ ) magnification. AMHR2 protein is stained green, and genetic material is stained blue (DAPI). Blue lines show the position of genetic material in the horizontal cuts of oocytes from above or from the side

In Additional file 6 and 7, box plots and Q-Q plots of AMHR2 gene expression in human oocytes obtained by RT-qPCR can be seen. The data comparing AMHR2 gene expression in oocytes matured under different conditions are presented in Additional file 8.

\section{Discussion}

The results of our study show for the first time that human oocytes express the protein and gene for AMHR2, the receptor for the hormone AMH. Moreover, in an IVM model, we found that AMH acts directly on oocytes and improves oocyte maturation in vitro.

In this study, in vitro matured oocytes were free of granulosa cells, which had been previously removed by oocyte denudation using hyaluronidase. It was confirmed that both AMHR2 protein and mRNA encoding it are expressed in human oocytes. Expressed mRNA along with the protein allows us to conclude that the oocytes probably synthesise AMHR2 on their own, which is a completely new finding.

AMHR2 protein was expressed in both immature and mature human oocytes, while the gene for this protein, $A M H R 2$, was expressed only in immature and in vitro matured oocytes. We speculate that the AMHR2 gene was expressed only in immature oocytes, as $A M H$ is needed for their maturation. After maturation, AMHR2 activity is probably no longer required, so the expression of this gene is stopped. However, the AMHR2 protein is probably still expressed in oocytes, as the receptors are not removed or recycled by the cell after maturation. Regardless, further research is needed.

The AMRH2 protein is thought to be located in the cell membrane as it is a membrane receptor [35]. Despite the fact that it is supposed to be a surface 


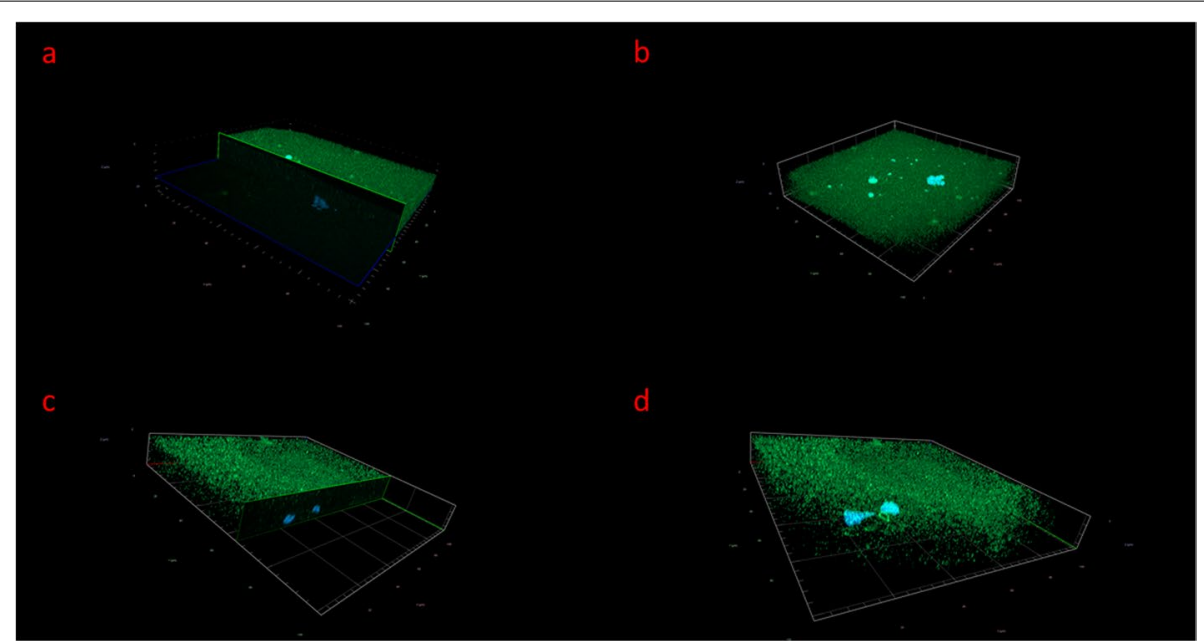

Fig. 3 Expression of AMHR2 protein (green) in human oocytes, as revealed by confocal microscopy. A Horizontal section of an AMHR2-positive immature oocytes. B Immature oocyte positively stained for AMHR2 protein. C Horizontal section of an AMHR2-positive mature oocyte. D Mature oocyte positively stained for AMHR2 protein. Images were taken with a confocal microscope at $630 \times$ magnification. AMHR2 is stained green, and genetic material is stained blue (DAPI)

antigen, we also performed permeabilization in the process of immunocytochemistry of oocytes. Permeabilization of the cell membrane is an extremely important step in detecting intracellular antigens with a primary antibody, because it allows entry across the cell membrane. Permeabilization is introduced after cells have been prepared with a fixative agent to initiate protein cross-linking. In our case, the fixative agent was paraformaldehyde. To permeabilize the membrane, we used a detergent Triton X-100 which permeabilizes the membrane by inserting a detergent monomer into the lipid membrane. This procedure was correct, as oocyte immunocytochemistry on AMHR2 was successfully performed. In addition, confocal microscopy in different planes showed that the AMHR2 protein is expressed throughout whole oocytes and not just on the surface as one would expect.

We performed IVM maturation of GV human oocytes under similar conditions to those of the study by Zhang et al. [35] in mouse oocytes. The addition of recombinant $\mathrm{AMH}$ alone to the IVM medium not only enabled immature oocytes to mature but also helped all the treated oocytes to mature (100\% maturation rate). This indicates that AMH directly affects human oocyte maturation in vitro and even improves it. In our study, the positive effect of recombinant $\mathrm{AMH}$ on the maturation of immature human oocytes in vitro was even greater than that of the commonly used hormones for IVM maturation - FSH and hCG. Perhaps this new knowledge can be used to further enhance the success of human oocyte IVM in the future, as this may mean






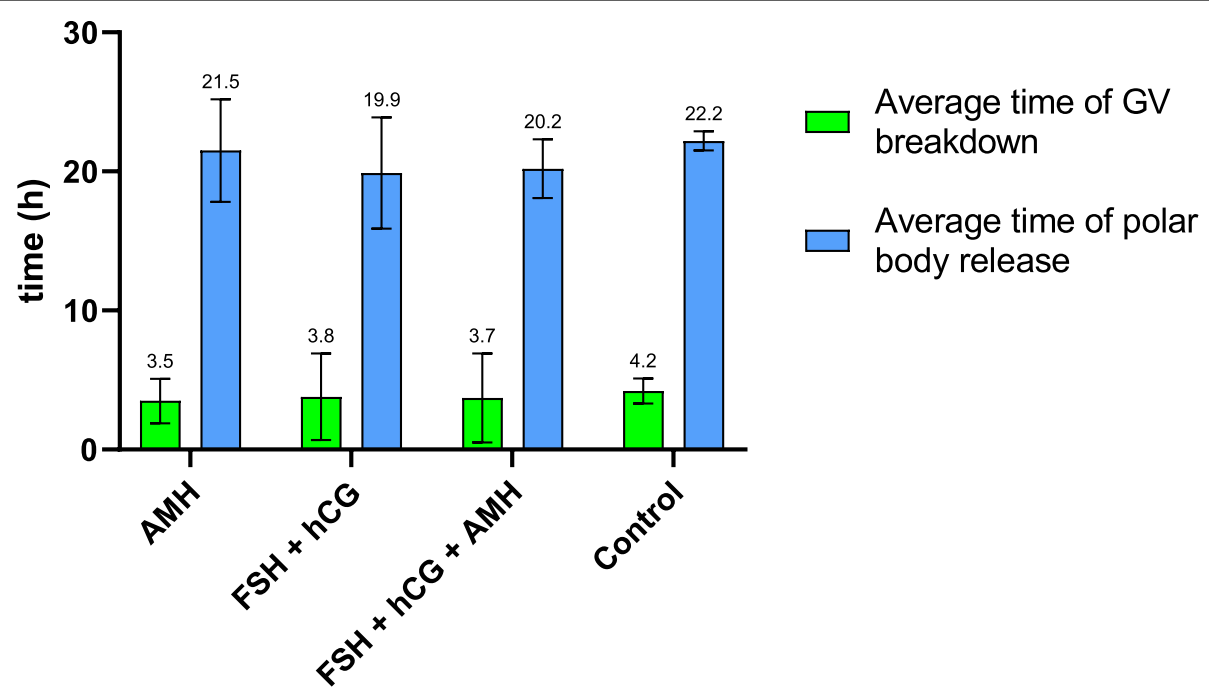

Fig. 5 Average time (hours) to GV breakdown and polar body release in oocytes matured in vitro. IVM medium containing one of the following combinations was used: i) $\mathrm{AMH}$, ii) FSH + hCG, iii) FSH + hCG + AMH, or iv) no added hormones (control). Data represents mean \pm SD. There was no significant difference between the groups (Student's t-test)



that the use of gonadotropins as a culture supplement is not needed or even not recommended in the procedure of IVM. Further research is needed with a higher number of oocytes, especially to test different concentrations of $\mathrm{AMH}$ added to the maturation medium, as well as to answer the question of whether maturation using recombinant $\mathrm{AMH}$ has an effect on the (epi) genetic status of oocytes.
$\mathrm{AMH}$ and FSH play antagonistic roles in human folliculogenesis [37, 38]. In this study, we observed that the addition of AMH, FSH and hCG to the maturation medium had a negative effect on oocyte maturation, which was significantly lower than that of IVM with AMH alone or FSH plus hCG. In contrast, Zhang et al. [35] used FSH and AMH together in IVM medium with no adverse effect on the maturation of mouse oocytes. 




Fig. 7 Average ratio of AMHR2 gene expression in mature oocytes compared to immature oocytes. Five different groups are presented. The first three are in vitro matured oocytes in IVM medium supplemented with i) $\mathrm{AMH}$, ii) FSH + hCG and iii) FSH $+\mathrm{hCG}+\mathrm{AMH}$. The last two groups represent untreated immature oocytes and in vivo mature oocytes. Data represents mean $\pm S D$. Statistical significance is shown at ${ }^{*} P<0.05$ (Kruskal-Wallis one-way analysis of variance)

Due to our findings, as also mentioned by certain researchers [37, 38], FSH and AMH have antagonistic actions and should not be used together in a maturation medium. It cannot be excluded that AMH and FSH have a different coaction depending on the species that is examined in vitro.

In comparison to Zhang et al. [35], we did not perform analysis on the expression of $\mathrm{AMH}$ in the cumulus cells as they were not the focus of this study and were removed during the oocyte preparation step. Despite this, we do agree that it is an important factor in trying to completely understand the function of $\mathrm{AMH}$ on the ovarian follicles and oocytes. Again, further research needs to be done on this manner, as it has the potential to change the clinical application of IVM in IVF cycles.

There are certain limitations to our study. The main limitation is that the number of oocytes used was fairly low. This is always the case when working with human oocytes, as they are hard to obtain in higher numbers. The proportion of immature oocytes after controlled ovarian hormonal stimulation is relatively low $(10-15 \%$ of all retrieved oocytes) [1,2]. Additionally, some of the GV oocytes also matured spontaneously, did not mature in vitro or degenerated during immunocytochemistry and were not included in the study.

\section{Conclusions}

In conclusion, we found that AMHR2 protein is expressed in both immature and mature human oocytes, which indicates that AMH can act on human oocytes directly. The $A M H R 2$ gene was expressed in immature and in vitro matured oocytes but not expressed in oocytes that matured in vivo in the ovaries. This further proves that AMH is important in the process of oocyte maturation, at least in vitro. Furthermore, the addition of only recombinant AMH to the maturation medium enabled and even improved the maturation of oocytes in vitro compared to the conventional IVM procedure with FSH and hCG in the maturation medium. The hormones AMH, FSH and hCG showed antagonism during oocyte maturation in vitro and should not be added to the maturation medium at the same time. Our findings prove that the current IVM protocol for immature human oocytes is not optimal and should be re-evaluated with the possibility of using recombinant $\mathrm{AMH}$ in the maturation medium. 


\section{Abbreviations}

AMH: Anti-Müllerian hormone; AMHR2: Anti-Müllerian hormone receptor 2; ART: Assisted reproductive technology; BMI: Body mass index; COCs: Cumulusoocyte complexes; CTCF: Corrected total cell fluorescence; FBS: Foetal bovine serum; FSH: Follicle-stimulating hormone; GnRH: Gonadotropin-releasing hormone; GV: Germinal vesicle; hCG: Human chorionic gonadotropin; ICSI: Intracytoplasmic sperm injection; IVF: In vitro fertilization; IVM: In vitro maturation; LH: Luteinizing hormone; OHSS: Ovarian hyperstimulation syndrome; PBS: Phosphate-buffered saline; PCOS: Polycystic ovaries syndrome; PGD: Preimplantation genetic diagnosis; POI: Primary ovarian insufficiency; RT-qPCR: Reverse-transcription quantitative real-time polymerase chain reaction.

\section{Supplementary Information}

The online version contains supplementary material available at https://doi. org/10.1186/s12958-022-00895-5.

Additional file 1. Western blot analysis of ascites cells from a patient with recurrent ovarian cancer for expression of AMHR2 protein, using old mouse (Ab64762) and new rabbit (Ab197148) anti-AMHR2 antibodies.

Additional file 2. Average age, BMI, FSH and LH levels of patients whose oocytes were used for IVM. Differences between groups were not statistically significant (ANOVA).

Additional file 3. The average age of donor women distributed between the groups of in vitro matured oocytes and immature or mature oocytes. There was no significant difference.

Additional file 4. Average cell fluorescence (ACF) measured in different groups of oocytes: immature, mature and control oocytes.

Additional file 5 . A $360^{\circ}$ video of a mature human oocyte that is positively stained for AMHR2 protein (green); genetic material is stained blue (DAPI). The video was generated using the confocal microscope.

Additional file 6. Box plot of RT-qPCR results for AMHR2 gene expression. Different groups of in vitro matured oocytes are represented with the median line and standard deviation.

Additional file 7. Q-Q plot of RT-qPCR results for AMHR2 gene expression. These plots show that all of the obtained results are fairly normally distributed despite the low number of samples. The outlier is the last group of in vivo matured oocytes, where no AMHR2 gene expression was observed. Because of this group, ANOVA was not possible, and KruskalWallis one-way analysis of variance had to be used.

Additional file 8. Comparisons of oocyte groups for AMHR2 gene expression. Kruskal-Wallis one-way analysis of variance was performed. There was a statistically significant difference in AMHR2 gene expression between the control groups (MII control vs. GV control, in yellow). Statistical significance was set at $P<0.05$.

\section{Acknowledgements}

Authors would like to thank all colleagues at the IVF Unit (gynaecologists, embryologists and nurses) of University Medical Centre Ljubljana, patients who kindly donated their oocytes for this research, and all others who contributed to this work.

\section{Authors' contributions}

Jure Bedenk: performed most of the experiments, performed most of the data analysis and wrote the manuscript. Tadeja Režen: performed part of the experiments (gene expression analysis) and part of the data analysis. Taja Železnik Ramuta: performed part of the experiments (confocal microscopy). Nina Jančar and Eda Vrtačnik Bokal: ovarian stimulation and oocyte collection. Ksenija Geršak: performed part of the experiments. Irma Virant Klun: idea, study design, performed part of the experiments, supervision. All authors contributed toward the drafting and revision of the paper and gave final approval of the version to be published.

\section{Funding}

The authors acknowledge the financial support from the Slovenian Research Agency (research core funding No. P3-0124, No. P1-0390, No. J3-2530 and young researcher scholarship for Jure Bedenk).

\section{Availability of data and materials}

The datasets used and analysed during the current study are available from the corresponding author on reasonable request.

\section{Declarations}

Ethics approval and consent to participate

Ethics were approved by the Republic of Slovenia National Medical Ethics Committee with the trial number 0120-546/2018/6. All participants in the study gave a written informed consent for using their oocytes in this study.

\section{Consent for publication}

Not applicable

\section{Competing interests}

The authors declare that they have no competing interests.

\section{Author details}

${ }^{1}$ Clinical Research Centre, University Medical Centre Ljubljana, 1000 Ljubljana, Slovenia. ${ }^{2}$ Institute of Biochemistry and Molecular Genetics, Centre for Functional Genomics and Bio-Chips, Faculty of Medicine, University of Ljubljana, 1000 Ljubljana, Slovenia. Institute of Cell Biology, Faculty of Medicine, University of Ljubljana, 1000 Ljubljana, Slovenia. ${ }^{4}$ Department of Gynaecology and Obstetrics, University Medical Centre Ljubljana, 1000 Ljubljana, Slovenia. ${ }^{5}$ Faculty of Medicine, University of Ljubljana, 1000 Ljubljana, Slovenia.

Received: 13 October 2021 Accepted: 16 January 2022

Published online: 24 January 2022

\section{References}

1. Cha K-Y, Chian R-C. Maturation in vitro of immature human oocytes for clinical use. Hum Reprod Update. 1998;4:103-20.

2. De Vos A, Van de Velde H, Joris H, Van Steirteghem A. In-vitro matured metaphase-l oocytes have a lower fertilization rate but similar embryo quality as mature metaphase-II oocytes after intracytoplasmic sperm injection. Hum Reprod. 1999;14:1859-63.

3. Ellenbogen A, Shavit T, Shalom-Paz E. IVM results are comparable and may have advantages over standard IVF. Facts Views Vis ObGyn. 2014;6:77-80.

4. Walls ML, Hart RJ. In vitro maturation. Best Pract Res Clin Obstet Gynaecol. 2018;53:60-72.

5. De Vos M, Grynberg M, Ho TM, Yuan Y, Albertini DF, Gilchrist RB. Perspectives on the development and future of oocyte IVM in clinical practice. $J$ Assist Reprod Genet. 2021;38:1265-80.

6. In vitro maturation. a committee opinion. Fertil Steril. 2021;115:298-304.

7. Thompson JG, Gilchrist RB. Improving oocyte maturation in vitro. In: Trounson A, Gosden R, Eichenlaub-Ritter U, editors. Biol pathol oocyte role fertil med nucl reprograming. 2nd ed. Cambridge: Cambridge University Press; 2013. p. 212-23.

8. Josso N, di Clemente N, Gouédard L. Anti-Müllerian hormone and its receptors. Mol Cell Endocrinol. 2001;179:25-32.

9. Kushnir VA, Seifer DB, Barad DH, Sen A, Gleicher N. Potential therapeutic applications of human anti-Müllerian hormone (AMH) analogues in reproductive medicine. J Assist Reprod Genet. 2017:34:1105-13.

10. Peluso C, Fonseca FLA, Rodart IF, Cavalcanti V, Gastaldo G, Christofolini $\mathrm{DM}$, et al. AMH: an ovarian reserve biomarker in assisted reproduction. Clin Chim Acta. 2014;437:175-82.

11. Rey R, Sabourin J-C, Venara M, Long W-Q, Jaubert F, Zeller WP, et al. Anti-Müllerian hormone is a specific marker of sertoli- and granulosa-cell origin in gonadal tumors. Hum Pathol. 2000;31:1202-8.

12. Visser JA, de Jong FH, Laven JSE, Themmen APN. Anti-Müllerian hormone: a new marker for ovarian function. Reproduction. 2006;131:1-9.

13. Nyström A, Mörse H, Nordlöf H, Wiebe K, Artman M, Øra I, et al. Anti-müllerian hormone compared with other ovarian markers after childhood cancer treatment. Acta Oncol. 2019:58:218-24.

14. AbdelHafez FF, Tang Y, Hassan MH, Saleem TH. Assessment of AntiMullerian Hormone (AMH) levels in a pilot cohort of peripubertal females: 
correlation with sex maturity rating (SMR). Middle East Fertil Soc J. 2018;23:278-80.

15. Sun H, Mao H, Cai J, Zhao Y. Research progress on anti-mullerian hormone clinical applications and immunoassay development. Front Lab Med. 2018;2:14-8.

16. Arce J-C, Nyboe Andersen A, Fernández-Sánchez M, Visnova H, Bosch E, García-Velasco JA, et al. Ovarian response to recombinant human folliclestimulating hormone: a randomized, antimüllerian hormone-stratified, dose-response trial in women undergoing in vitro fertilization/intracytoplasmic sperm injection. Fertil Steril. 2014;102:1633-1640.e5.

17. Wang JG, Douglas NC, Nakhuda GS, Choi JM, Park SJ, Thornton MH, et al. The association between anti-Müllerian hormone and IVF pregnancy outcomes is influenced by age. Reprod Biomed Online. 2010;21:757-61.

18. Sahmay S, Oncul M, Tuten A, Tok A, Acıkgoz AS, Cepni I. Anti-Müllerian hormone levels as a predictor of the pregnancy rate in women of advanced reproductive age. J Assist Reprod Genet. 2014;31:1469-74.

19. Webber L, Stubbs S, Stark J, Trew G, Margara R, Hardy K, et al. Formation and early development of follicles in the polycystic ovary. The Lancet. 2003;362:1017-21.

20. Jahromi BN, Dabbaghmanesh MH, Bakhshaie P, Parsanezhad ME, Anvar Z, Alborzi M, et al. Assessment of oxytocin level, glucose metabolism components and cutoff values for oxytocin and anti-mullerian hormone in infertile PCOS women. Taiwan J Obstet Gynecol. 2018;57:555-9.

21. Rashad NM, Moafy H, Saleh HS, Amin Al, Gomaa AF. Anti-Müllerian hormone: predictor of premature ovarian insufficiency in Egyptian women with autoimmune thyroiditis. Middle East Fertil Soc J. 2018;23:286-91.

22. Kim C, Slaughter JC, Wang ET, Appiah D, Schreiner P, Leader B, et al. AntiMüllerian hormone, follicle stimulating hormone, antral follicle count, and risk of menopause within 5 years. Maturitas. 2017;102:18-25.

23. Färkkilä A, Koskela S, Bryk S, Alfthan H, Bützow R, Leminen A, et al. The clinical utility of serum anti-Müllerian hormone in the follow-up of ovarian adult-type granulosa cell tumors - a comparative study with inhibin B. Int J Cancer. 2015;137:1661-71.

24. Chong YH, Campbell AJ, Farrand S, McLennan IS. Anti-Müllerian hormone level in older women: detection of granulosa cell tumor recurrence. Int J Gynecol Cancer. 2012;22:1497-9.

25. Ge W, Clendenen TV, Afanasyeva Y, Koenig KL, Agnoli C, Brinton LA, et al. Circulating Anti-Müllerian hormone and breast cancer risk: a study in ten prospective cohorts. Int J Cancer. 2018;142:2215-26.

26. Baarends WM, van Helmond MJ, Post M, van der Schoot PJ, Hoogerbrugge JW, de Winter JP, et al. A novel member of the transmembrane serine/threonine kinase receptor family is specifically expressed in the gonads and in mesenchymal cells adjacent to the müllerian duct. Dev Camb Engl. 1994;120:189-97.

27. di Clemente N, Wilson C, Faure E, Boussin L, Carmillo P, Tizard R, et al. Cloning, expression, and alternative splicing of the receptor for antiMüllerian hormone. Mol Endocrinol Baltim Md. 1994;8:1006-20.

28. Pfennig F, Standke A, Gutzeit HO. The role of amh signaling in teleost fish - multiple functions not restricted to the gonads. Gen Comp Endocrinol. 2015;223:87-107.

29. Gouédard L, Chen Y-G, Thevenet L, Racine C, Borie S, Lamarre I, et al. Engagement of bone morphogenetic protein type IB receptor and smad1 signaling by Anti-Müllerian hormone and its type II receptor*. J Biol Chem. 2000;275:27973-8.

30. Racine C, Rey R, Forest MG, Louis F, Ferré A, Huhtaniemi I, et al. Receptors for anti-müllerian hormone on leydig cells are responsible for its effects on steroidogenesis and cell differentiation. Proc Natl Acad Sci U S A. 1998:95:594-9

31. Ingraham HA, Hirokawa Y, Roberts LM, Mellon SH, McGee E, Nachtigal MW, et al. Autocrine and paracrine müllerian inhibiting substance hormone signaling in reproduction. Recent Prog Horm Res. 2000;55:53-67 (discussion 67-68)

32. Segev DL, Hoshiya Y, Hoshiya M, Tran TT, Carey JL, Stephen AE, et al. Mullerian-inhibiting substance regulates NF-KB signaling in the prostate in vitro and in vivo. Proc Natl Acad Sci. 2002;99:239-44.

33. Segev DL, Hoshiya Y, Stephen AE, Hoshiya M, Tran TT, MacLaughlin DT, et al. Müllerian inhibiting substance regulates NFkB signaling and growth of mammary epithelial cells in vivo. J Biol Chem. 2001;276:26799-806.

34. Wang J, Dicken C, Lustbader JW, Tortoriello DV. Evidence for a Müllerianinhibiting substance autocrine/paracrine system in adult human endometrium. Fertil Steril. 2009:91:1195-203.
35. Zhang Y, Shao L, Xu Y, Cui Y, Liu J, Chian R-C. Effect of Anti-Mullerian Hormone in culture medium on quality of mouse oocytes matured in vitro. PLOS One. 2014;9:e99393.

36. Saleh AC, Sabry R, Mastromonaco GF, Favetta LA. BPA and BPS affect the expression of anti-Mullerian hormone (AMH) and its receptor during bovine oocyte maturation and early embryo development. Reprod Biol Endocrinol. 2021:19:119.

37. Dewailly D, Robin G, Peigne M, Decanter C, Pigny P, Catteau-Jonard S. Interactions between androgens, FSH, anti-Müllerian hormone and estradiol during folliculogenesis in the human normal and polycystic ovary. Hum Reprod Update. 2016;22:709-24.

38. Visser JA, Themmen APN. Anti-Müllerian hormone and folliculogenesis. Mol Cell Endocrinol. 2005;234:81-6.

39. Virant-Klun I, Leicht S, Hughes C, Krijgsveld J. Identification of maturationspecific proteins by single-cell proteomics of human oocytes. Mol Cell Proteomics MCP. 2016;15:2616-27.

40. Schindelin J, Arganda-Carreras I, Frise E, Kaynig V, Longair M, Pietzsch $\mathrm{T}$, et al. Fiji - an open source platform for biological image analysis. Nat Methods. 2012;9:676-82.

41. Gavet O, Pines J. Progressive activation of cyclinb1-cdk1 coordinates entry to Mitosis. Dev Cell. 2010;18:533-43.

42. Andersen CL, Jensen JL, Ørntoft TF. Normalization of real-time quantitative reverse transcription-PCR data: a model-based variance estimation approach to identify genes suited for normalization, applied to bladder and colon cancer data sets. Cancer Res. 2004;64:5245-50.

43. Livak KJ, Schmittgen TD. Analysis of relative gene expression data using real-time quantitative PCR and the 2(-Delta Delta $C(T))$ method. Methods San Diego Calif. 2001;25:402-8.

44. The jamovi project. jamovi (Version 1.6). 2021. Available from: https:// jamovi.org

\section{Publisher's Note}

Springer Nature remains neutral with regard to jurisdictional claims in published maps and institutional affiliations.

Ready to submit your research? Choose BMC and benefit from

- fast, convenient online submission

- thorough peer review by experienced researchers in your field

- rapid publication on acceptance

- support for research data, including large and complex data types

- gold Open Access which fosters wider collaboration and increased citations

- maximum visibility for your research: over $100 \mathrm{M}$ website views per year

At BMC, research is always in progress.

Learn more biomedcentral.com/submissions 